\title{
SECOND-ORDER EFFECTS DUE TO ROTATION IN PULSATINC DA WHITE DWARFS
}

\author{
P. Brassard, F. Kesemael, and G. Fontaine. \\ Département de Physique, Université de Montréal
}

The $Z Z$ Ceti star $L$ 19-2 is a stable pulsator whose light curve has now been deciphered with the help of over 300 hours of white light, high-speed photometry (ODonoghue and Warner 1982, 1987, hereafter ODW). The analysis indeed reveals the presence in the light curve of five coherent oscillations, with periods ranging from $113 \mathrm{~s}$ to $350 \mathrm{~s}$. Among those, the $192 \mathrm{~s}$ oscillation possesses three components, almost equally separated in frequency. Most importantly, the slight, but statistically significant, inequality in the frequency spacing of the triplet has been interpreted by these authors as second-order splitting of rotationally-perturbed g-mode oscillations. And indeed, the measured splitting appears consistent with the theoretical predictions of Chlebowski (1978), which are based on somewhat archaic white dwarf models. As pointed out by ODY, it is clearly of great interest to investigate 1) to what extent theoretical predictions based on more realistic, current-generation white dwarf models agree with ODW's identification, and 2) to what extent such second order effects can, eventually, be used to identify individual pulsation modes or constrain the structural parameters of variable white dwarf stars. Motivated by these questions, have initiated a study of second-order effects due to rotation in $Z Z$ Ceti stars, and we report here the first results of this program.

The perturbed state of a star undergoing non-radial oscillations can be written as a superposition of eigenmodes, each characterized by one eigenfrequency and three quantum numbers: $k$, the radial wavenumber, and $I$ and $m$ the spherical harmonics indices which describe the angular geometry. For a non-rotating star, the eigenfrequencies are degenerate in $m$, and depend only on $k$ and 1 . In the presence of slow rotation $\left(\Omega \ll o_{k l}\right)$ we can write, for an external observer 


$$
o_{k \mid m}=o_{k l}+m\left(1-C_{k l}\right) \Omega+O\left[\Omega^{2}\right)
$$

Where $C_{k l}$ is a function of the structure of the star and of the particular eigenmode. The pulsation mode is now split in $21+1$ equally-spaced frequencies. A nice example of this splitting is the 109.28 triplet in G226-29, with observed frequencies of $9.134721,9.150865$, and $9.167009 \mathrm{mHz}$ separated by $0.016144 \mathrm{mHz}$ (Kepler, Robinson, and Nather 1983).

It is also possible to expand equation (1) to include second-order terms (Chlebowski 1978; Saio 1981; Smeyers and Martens 1983). We write, following Chlebowski's notation

$$
o_{k \mid m}=o_{k 1}+m\left(1-C_{k 1}\right) \Omega+\frac{1}{2 o_{k 1}}\left(P_{k 1}-m^{2} Q_{k 1}\right) \Omega^{2}+O\left(\Omega^{3}\right)
$$

For large-k modes in white dwarfs, the asymptotic values of $P$ and $Q$ are given by

$$
P=\frac{4[2 \mid(\mid+1)-3]}{(2 \mid-1)(2 \mid+3)} \text { and } Q=\frac{4 \mid(\mid+1)\left(\left.2\right|^{2}+2 \mid-3\right)-9}{I^{2}(1+1)^{2}(2 \mid-1)(2 \mid+3)}
$$

The approach of Saio (1981) is used to calculate $P$ and $Q$.

Equations (1) and (2) are valid only if solid body rotation is assumed for the star. If differential rotation is included, equation (1) should be rewritten (Hansen, Cox, and Van Horn 1977)

$$
o_{k \mid m}-o_{k \mid}+m\left(1-C_{k \mid}-C_{k|| m \mid}^{\prime}\right) \Omega
$$

We ignore departures from solid body rotation here, as Tassoul and Tassoul (1983) have shown that such departures are likely to be small in white dwarfs. In addition, we leave out as well the perturbations to the eigenfrequencies that could be caused by a magnetic field, as recently shown by Jones et al. (1988). 
We have thus adapted Saio's (1981) formalism, developed for a polytropic star, to the case of more realistic stellar configurations. Furthermore, to evaluate the second order terms, the distorted geometry of the star caused by rotation is first required. To this end, used a Clairaut-Legendre expansion (80e, for example, Tassoul 1979), although more elaborate methods are possible. Sample calculations of the first- and second-order splitting terms (C,P and $Q$, respectively) are presented in Table 1 for a model at $0.8 \mathrm{M}_{\odot}$, with a hydrogen (helium) fractional layer mass of $10^{-12}\left(10^{-2}\right)$ and an effective temperature of $11,519 \mathrm{~K}$, taken from the extensive evolutionary sequences of Tassoul, Fontaine, and Winget [1988).

\section{TABLE 1}

\begin{tabular}{|c|c|c|c|c|c|}
\hline \multicolumn{6}{|c|}{$\begin{array}{c}\text { First- and Second-order Splitting Terms } \\
M_{0}=0.8, T_{0}=11,519 \mathrm{~K}\end{array}$} \\
\hline 1 & $k$ & Period(s) & $C_{k 1}$ & $P_{k 1}$ & $Q_{k 1}$ \\
\hline $\begin{array}{l}1 \\
1 \\
1 \\
1 \\
1 \\
1 \\
1\end{array}$ & $\begin{array}{l}1 \\
2 \\
3 \\
4 \\
5 \\
6 \\
\infty\end{array}$ & $\begin{array}{l}222.4 \\
270.4 \\
291.0 \\
371.0 \\
394.9 \\
455.8 \\
\end{array}$ & $\begin{array}{l}0.457 \\
0.270 \\
0.458 \\
0.444 \\
0.436 \\
0.484 \\
0.500\end{array}$ & $\begin{array}{l}0.868 \\
1.174 \\
0.873 \\
0.895 \\
0.909 \\
0.828 \\
0.800\end{array}$ & $\begin{array}{r}-0.039 \\
0.063 \\
0.185 \\
-0.073 \\
0.126 \\
-0.035 \\
-0.050\end{array}$ \\
\hline $\begin{array}{l}2 \\
2 \\
2 \\
2 \\
2\end{array}$ & $\begin{array}{l}1 \\
2 \\
3 \\
4 \\
\infty\end{array}$ & $\begin{array}{l}129.2 \\
165.8 \\
191.9 \\
219.7\end{array}$ & $\begin{array}{l}0.134 \\
0.144 \\
0.004 \\
0.151 \\
0.166\end{array}$ & $\begin{array}{l}1.751 \\
1.741 \\
1.901 \\
1.734 \\
1.714\end{array}$ & $\begin{array}{l}0.270 \\
0.249 \\
0.293 \\
0.279 \\
0.274\end{array}$ \\
\hline
\end{tabular}

Similar calculations were performed for a number of models culled from several evolutionary sequences of varying stellar mass, and hydrogen and helium layer masses in order to compare the predicted second-order splitting with that measured by ODW. We summarize, below, the observed structure of the 1928 mode in $L$ 19-2. 
TABLE 2

\begin{tabular}{|c|c|c|c|}
\hline \multicolumn{4}{|c|}{ Observed Structure of the $192 \mathrm{~s}$ oscillation in L19-2 } \\
\hline Period (s) & Frequency (mHz) & $\Delta v(\mathrm{mHz})$ & $\varepsilon(\mathrm{mHz})$ \\
\hline 192.128824 & 5.2048411 & 0.0129912 & \\
192.609575 & 5.19184989 & 0.0129931 & $-(1.9 \pm 0.4) \times 10^{-6}$ \\
193.092808 & 5.1788568 & & \\
\hline
\end{tabular}

On the basis of equation (2), the measured second-order splitting $\varepsilon$ can be related to the $Q$ coefficient by

$$
Q=-\varepsilon v_{0}\left[\frac{\Delta m\left(1-C_{k 1}\right)}{\Delta v}\right]^{2}
$$

For a $1=1$ mode, the value of $C$ is of the order of $C=0.4 \pm 0.1$ (see Table 1) and thus the ODW measurement yields $Q=0.02 \pm 0.01$. A comparison with the $Q$-values displayed in Table 1 suggests that small, positive values are possible only for some low-k modes. Furthermore, a period as short as $192 \mathrm{~s}$ for $1-1$ can only be reproduced for low-k values $(k=1$ or 2$)$ if the stellar mass is $0.8 M_{\odot}$ and both the helium and hydrogen layer masses are small. These constraints are summarized in Table 3 . For a given model, the range of values of $k$ corresponding to $Q>0$ is indicated, as well as the minimal period (minimal $k$ ). The results suggest that the range of allowed values of $k$ decreases with decreasing $H$ and $H e$ outer layer masses, and that the minimal period decreases as well. If the $192 \mathrm{~s}$ oscillation of $L 19-2$ is to be associated with a 1-1 mode, the star must have a higher than average gravity for a white dwarf. This is amenable to abservational verification. At the same time, however, this solution is by no means unique; indeed our analysis suggests that both the Qmeasurement and the observed period might also be consistent with a $1=3$ mode in a DA star at $M \geqslant 0.6 M_{\odot}$ with thick $\left(M_{H e} 310^{-2}\right)$ helium layers. This would require that only the components with $m-0, \pm 1$ of the $192 \mathrm{~s}$ mode and $m=0, \pm 3$ of the $113 \mathrm{~s}$ mode be 
observable. Clearly, we have only scratched the surface, and the potential of this new tool for the study of L 19-2, and ZZ Ceti stors in general, has barely been tapped.

TABLE 3

\begin{tabular}{|c|c|c|c|c|}
\hline \multicolumn{3}{|c|}{ Modes with $Q \geqslant 0(1-1)$, in Var ious Stellar Models } \\
\hline Mass & $\log \left(M_{\text {He }}\right)$ & $\log \left(M_{H}\right)$ & $k=123456789$ & $\begin{array}{c}\text { Minimal } \\
\text { Per iod (s) }\end{array}$ \\
\hline 0.6 & -2 & -6 & & 315 \\
0.6 & -2 & -10 & & 330 \\
0.6 & -2 & -12 & & 300 \\
0.6 & -6 & -10 & & 280 \\
0.6 & -6 & -12 & & 280 \\
\hline 0.8 & -2 & -6 & & 250 \\
0.8 & -2 & -10 & & 265 \\
0.8 & -2 & -12 & & 235 \\
0.8 & -6 & -12 & & \\
\hline
\end{tabular}

We are grateful to B. Wamer for drawing our attention to and triggering our interest in this remarkable observational result, and to SD. Kawaler, M. Tassoul and P. Smeyers for useful discussions. This work was supported in port by the NSERC Canada, by the Fund FCAR (Québec), and by a E.WR. Steacie Memorial Fellowship to one of us (GF).

\section{REFERENCES}

Chiebowski, T. 1978, Acta Astr., 24, 441.

Honsen, C.J., Cox, J.P., and Von Horn, HM. 1977, A. J., 217, 151.

Jones, P.W, Pesnall, WD., Hansen, C.J., and Kowaler, SD. 1989, Preprint.

Kepler, S.0., Rabinson, EL., and Nother, RE. 1983, Ao. J., 271, 744.

O'Donoghwe, D., and Warner, B. 1982, N.NR.A.S., 133, 293.

O'Donoghwe, D., and Worner, B. 1987, M.N.R.A.S., 228, 949.

Saio, H. 1981, 10. A, 244, 299.

Smeyers, P., and Martens, L. 1983, Astr. 10., 120, 193.

Tossoul, J1. 1979, The Theory of Rotating Stars, (Princeton: Princeton University Press), p. 96.

Tassoul, M., Fontaine, G., and Winget, DE. 1989, in preparation.

Tassoul, M., and Tassoul, J.L. 1983, Ap. J., 237, 334. 